\title{
Transcranial Doppler as a Bedside Evaluation Tool in Tubercular Meningitis: Case Report and Review of Literature
}

\author{
Dhananjay Gupta, Pradeep R*, Anjani Kumar Bhushan, Anish Mehta, Mahendra Javali, \\ Purshottam T Acharya, Rangasetty Srinivasa \\ Department of Neurology, M S Ramaiah Medical College, Bengaluru, India
}

*Corresponding Author: Pradeep R, Department of Neurology, M S Ramaiah Medical College, Bengaluru, India, Email: dhananjay_gupta1990@yahoo.com

\begin{abstract}
Transcranial Doppler (TCD) is an emerging modality for bedside evaluation of cerebral hemodynamics. It provides an easy, convenient and non-invasive modality for the assessment of intracranial blood flows and pressure. Role of TCD in evaluation and management of vasculopathy related to tubercular meningitis (TBM) has shown contrasting results in previous studies We describe the TCD findings in a patient of TBM with extensive basal exudates, which were reversible after treatment with antitubercular therapy (ATT) and steroids.
\end{abstract}

Keywords: Transcranial doppler; Tubercular meningitis;, TBM vasculopathy; Vasculitic infarcts; Steroids

\section{INTRODUCTION}

Vasculopathy is a well-recognized and frequently catastrophic complication of Tubercular Meningitis (TBM). ${ }^{1}$ The hallmark feature is development of basal exudates with cranial nerve palsies or cerebral infarctions. ${ }^{2}$ Extensive damage to cerebral vessels causes widespread infarctions, including brainstem involvement. ${ }^{3,4} \quad$ Transcranial doppler ultrasonography (TCD) provides a safe, efficacious, non-invasive bedside method of evaluation of arteries at the base of the brain. ${ }^{5}$ Critically ill patients with advanced disease may require frequent close monitoring of cerebral hemodynamics, including intracranial blood flows and pressures. Repetitive evaluations with Magnetic Resonance Imaging (MRI) or Lumbar puncture may be difficult and thus TCD may play an important role in the management of these patients. Here we report a case of TBM with vasculopathy who showed elevated blood flow velocities in the Posterior (PCA), Middle (MCA) and Anterior Cerebral Arteries (ACA). These changes were reversible after treatment with ATT and steroids.

\section{CASE History}

43-year-old lady, without comorbidities, complained of intermittent fever associated with holocranial throbbing headache for 3 months and irrelevant speech for 5 days prior to presentation. She was drowsy, arousable to verbal stimuli and was able to follow simple commands. Vitals were stable and she had neck stiffness. Cranial nerves, eye, motor and sensory examination were grossly normal. Cerebellar examination was remarkable for limb ataxia. Blood investigations showed high Erythrocyte Sedimentation Rate (ESR) of 42. Complete blood counts, electrolytes, renal and liver functions were normal. A Magnetic Resonance imaging of the brain showed T2/ FLAIR hyperintensities in bilateral brainstem, right temporal lobe and left centrum semi-ovale with diffusion restriction, suggestive of acute infarcts. (Figure 1) A post-contrast study revealed basal exudates with pachymeningeal enhancement, consistent with tubercular meningitis. A lumbar puncture showed high opening pressure of $30 \mathrm{~cm}$ of water with Cerebro-spinal fluid (CSF) analysis showing 45 cells (70\% lymphocytes) with high protein- $800 \mathrm{mg} / \mathrm{dL}$ and CSF sugar of $45 \mathrm{mg} / \mathrm{dL}$. CSF gram stain, culture, $\mathrm{KOH}$ mount, testing for cryptococcal antigen and India ink preparation were negative. A CSF meningoencephalitis panel, including testing for neuro viral infections was negative. CSF testing for tuberculosis with Polymerase chain reaction (TB-PCR) was positive. A bedside TCD study, using a $2 \mathrm{MHz}$ probe, at the time of presentation showed high mean and peak systolic velocities in the bilateral Middle cerebral artery (Right $120 \mathrm{~cm} / \mathrm{sec}$, left- $138 \mathrm{~cm} / \mathrm{sec}$ ) and bilateral posterior cerebral artery (right P1 $-109 \mathrm{~cm} / \mathrm{sec}$, 
left P1 - $157 \mathrm{~cm} / \mathrm{sec})$. Anterior cerebral artery (ACA) velocities were $82.8 \mathrm{~cm} / \mathrm{sec}$ on the right and $119 \mathrm{~cm} / \mathrm{sec}$ on the left. (Figure 2)

Corresponding PI values in Right MCA was 0.63 , in left MCA was 0.71 , in right ACA - 1.04 and in left ACA was 1.43. She was initiated on anti-tubercular therapy along with intravenous steroids. She showed symptomatic improvement in sensorium and headache after 3 days of initiation of steroids. On day 12, repeat MRI brain showed resolution of basal exudates. This correlated with improved TCD measures, in terms of reduced peak systolic velocity in the right MCA to $100 \mathrm{~cm} / \mathrm{sec}$, in left MCA to 79.7 $\mathrm{cm} / \mathrm{sec}$, in right ACA to 50.8, left ACA to 102, in right PCA-P1 to 77 and left PCA-P1 to 68.1 $\mathrm{cm} / \mathrm{sec}$. Similar results were seen in the peak velocities of Basilar artery. (Figure 2) Mean flow velocities in all intracranial vessels showed a similar decreasing trend after treatment with steroids. The PI values did not show any significant reduction in the Middle or a posterior cerebral artery, however, a decrease in PI values was noted in the Basilar artery.

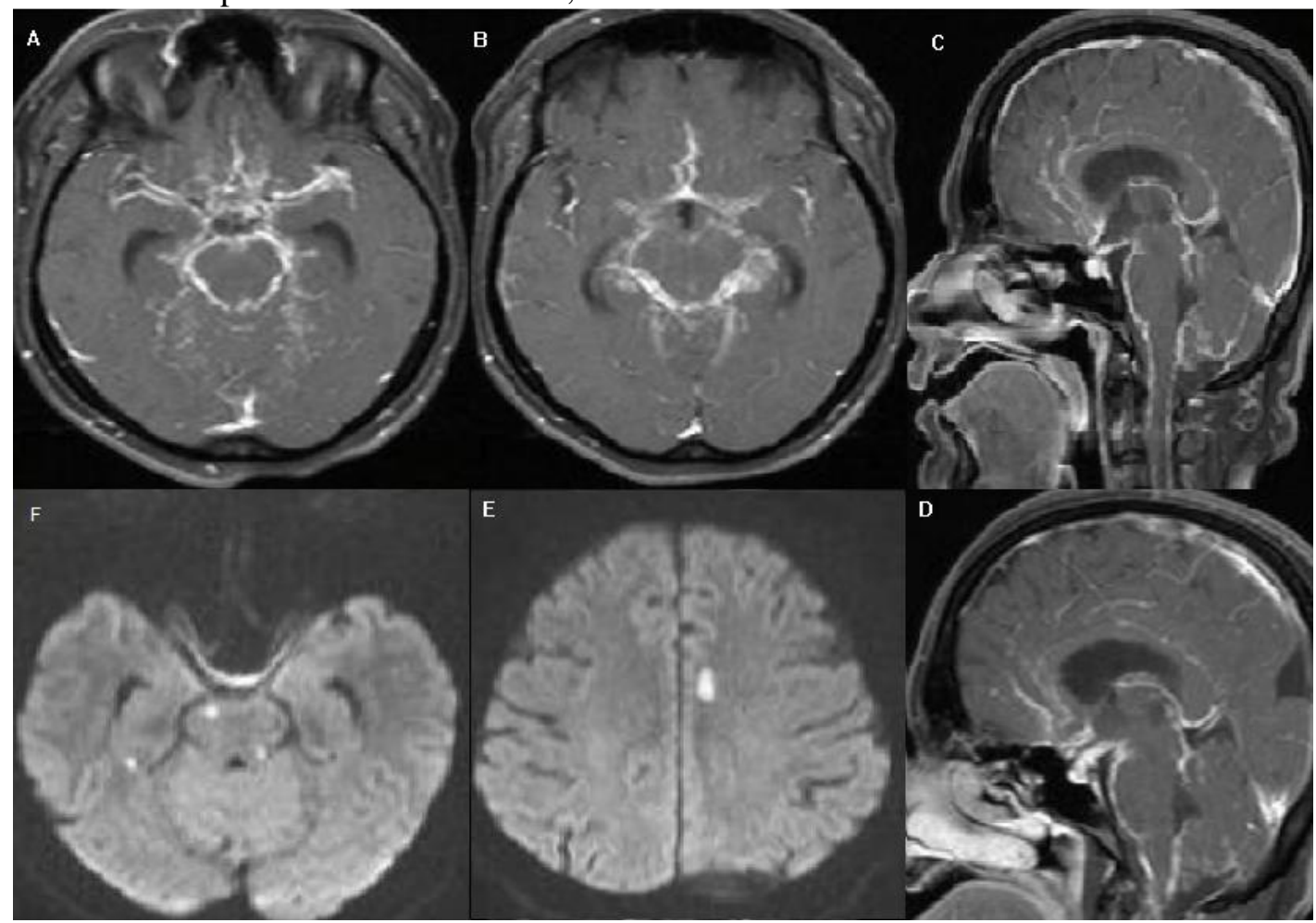

Figure1: MRI brain with contrast showing extensive meningeal enhancement and basal exudates (a,b: axial cuts and c,d: sagittal cuts). Diffusion weighted images showing acute vasculitic infarcts in the bilateral midbrain, right temporal region and left cerebral cortex.

\section{DISCUSSION}

TCD is an inexpensive and sensitive measure of intracranial blood flow velocities, which helps in identifying areas of arterial stenosis. In addition, it also helps in monitoring the management of raised intracranial pressures (ICP) in TBM patients. Tai et. al. correlated TCD findings in 36 patients of TBM with Computed Tomography or Magnetic Resonance angiography (CTA/ MRA). $80 \%$ of patients having TCD criteria for vasculopathy, showed focal narrowing of major intracranial arteries on either CTA/MRA. ${ }^{6}$ Van-Toorn et. al. used TCD to study blood flow velocities and found high velocities in basal cerebral arteries in 14 children diagnosed with TBM. $^{7}$ These high values persisted for 7 days suggesting the cause of elevated velocities to be vasculitis rather than functional vasospasm. Further, in children with non-communicating hydrocephalus, a decline in PI values was noted post CSF diversion procedure.

However, similar results were not seen in children with communicating hydrocephalus, who showed a poor correlation between PI values and ICP. In another study, children having TBM with hydrocephalus with vasculopathy and cerebral infarcts, CSF diversion procedures failed to decrease the PI values. Post-operative PI values in these patients were significantly higher as compared to children having TBM with hydrocephalus but without infarcts 8 . 


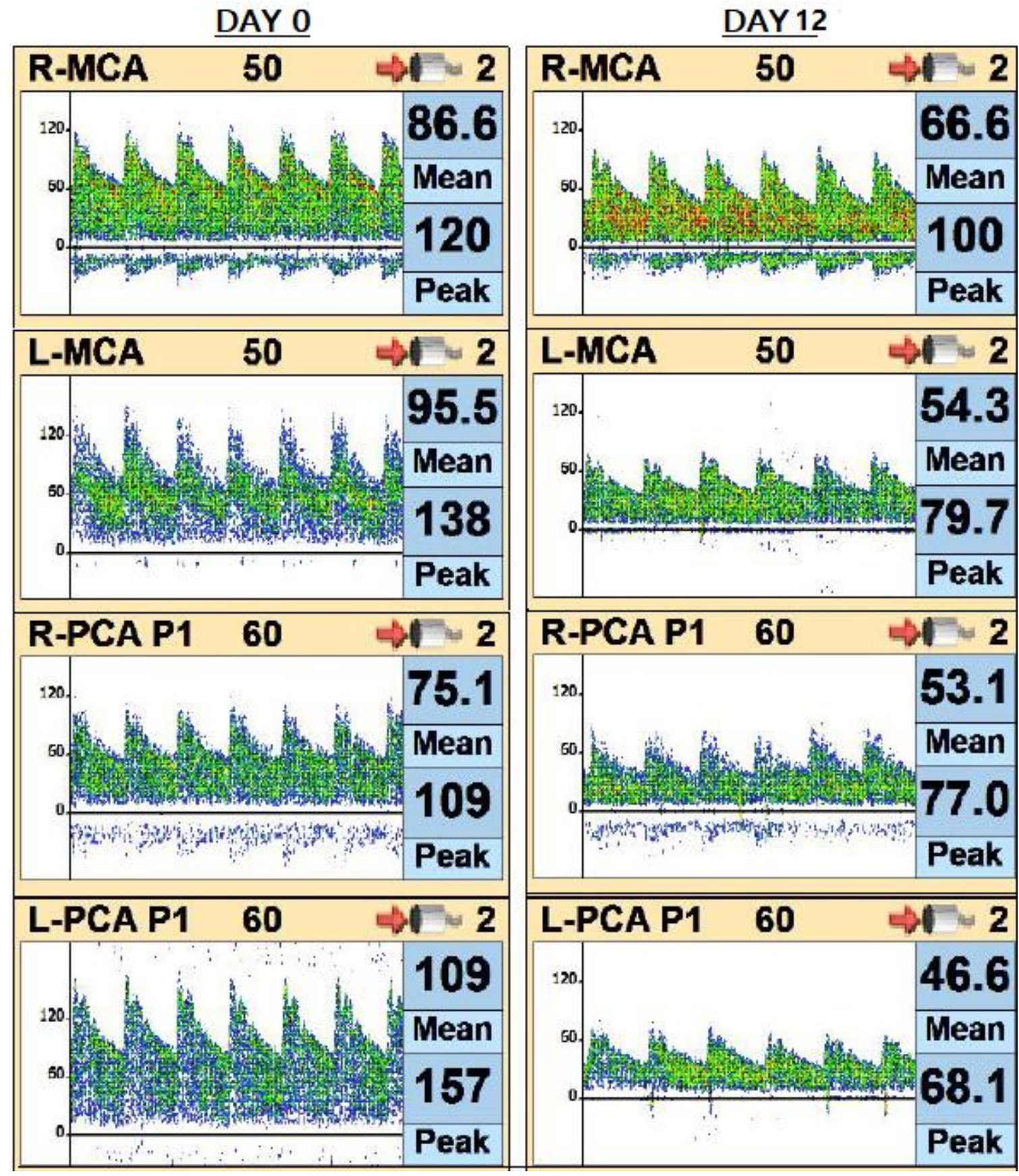

Figure2: Transcranial doppler findings on Day 0 (before starting treatment) and Day 12 (after 12 days of therapy with ATT and steroids).

In our patient, initial TCD assessment at the time of presentation showed high peak systolic and mean flow velocities in the insonated intracranial vessels, which correlated with dense basal exudates and pachymeningeal enhancement on MR imaging. Based on the criteria proposed by various authors, ${ }^{6,-11}$ TCD values indicated Left PCA vasculopathy (MFV> $85 \mathrm{~cm} / \mathrm{sec}$ ) with $>50 \%$ stenosis (PSV >145 $\mathrm{cm} / \mathrm{sec}$ ) and $<50 \%$ narrowing in right PCA (PSV 100-145 cm/sec). After treatment with steroids, the basal exudates settled as shown in repeat MRI, and TCD showed reduced peak and mean flow velocities, signifying reduced obstruction to blood flow in the tested intracranial vessels.

To conclude, patients with TBM-vasculopathy develop cerebral hemodynamic alterations due to inflammatory lesions in the subarachnoid space leading to steno-occlusive disease of the cerebral vessels. This can be assessed via a bedside Transcranial doppler, which helps in identifying vascular narrowing, diagnosing vasculopathy and monitoring the response to therapy. 


\section{REFERENCES}

[1] Wasay M, Farooq S, Khowaja ZA, Bawa ZA, Ali SM, Awan S, et. al. Cerebral infarction and tuberculoma in central nervous system tuberculosis: frequency and prognostic implications. J NeurolNeurosurg Psychiatry. 2014; 85(11): 1260-1264.

[2] Corr PD. Imaging in CNS Tuberculosis. Medscape. Accessed 20 ${ }^{\text {th }}$ December 2018. Available from: https://emedicine.medscape. com/article/344862-overview

[3] Chatterjee D, Radotra BD, Vasishta RK, Sharma K. Vascular complications of tuberculous meningitis: An autopsy study. Neurol India. 2015; 63(6):926-32.

[4] Jagetia A. Tuberculous vasculitis: The neurosurgeon's perspective. Neurol India. 2016; 64(5): 868-70.

[5] Kiliç T, Elmaci I, Özek MM, Pamir NM. Utility of transcranial Doppler ultrasonography in the diagnosis and follow-up of tuberculous meningitis-related vasculopathy. Childs Nerv Syst. 2002; 18(3-4): 142-146.
[6] Tai MS, Sharma VK. Role of Transcranial Doppler in the Evaluation of Vasculopathy in Tuberculous Meningitis. PLoS One. 2016; 11 (10): e0164266.

[7] Van Toorn R, Schaaf HS, Solomons R, Laubscher JA, Schoeman JF. The value of transcranial Doppler imaging in children with tuberculous meningitis. Childs Nerv Syst. 2014; 30(10): 1711-1716.

[8] Nadvi SS, Van-dellen J, Gouws E. Transcranial doppler ultrasonography in hydrocephalic children with tuberculous meningitis. $\mathrm{Br} J$ Neurosurg. 1995; 9(4): 519-26.

[9] Lu CH, Chang HW, Lui CC, Huang CR, Chang WN. Cerebral haemodynamics in acute bacterial meningitis in adults. QJM. 2006; 99(12): 863-869.

[10] Baumgartner RW, Mattle HP, Schroth G. Assessment of $>/=50 \%$ and $<50 \%$ intracranial stenoses by transcranial color-coded duplex sonography. Stroke. 1999; 30(1): 87-92.

[11] Müller M, Merkelbach S, Schimrigk K. Cerebral hemodynamics in the posterior circulation of patients with bacterial meningitis. Acta Neurol Scand. 1996; 93(6):443-9

Citation: Dhananjay Gupta, Pradeep R, et al. N Transcranial Doppler as a Bedside Evaluation Tool in Tubercular Meningitis: Case Report and Review of Literature. ARC Journal of Neuroscience. 2019; 4(1):27-30. doi: dx.doi.org/10.20431/2456-057X 0401004.

Copyright: (c) 2019 Authors. This is an open-access article distributed under the terms of the Creative Commons Attribution License, which permits unrestricted use, distribution, and reproduction in any medium, provided the original author and source are credited. 